\title{
MAC Protocol for Opportunistic Cognitive Radio Networks with Soft Guarantees
}

\author{
Haythem Bany Salameh and Marwan Krunz \\ Department of Electrical and Computer Engineering \\ University of Arizona, Tucson, AZ 85721 \\ Email: \{haythem,krunz\}@ece.arizona.edu
}

\author{
Ossama Younis \\ Applied Research, Telcordia Technologies, Inc. \\ Piscataway, NJ 08854 \\ Email: oyounis@ research.telcordia.com
}

\begin{abstract}
Cognitive radio (CR) is the key enabling technology for an efficient dynamic spectrum access. It aims at exploiting an underutilized licensed spectrum by enabling opportunistic communications for unlicensed users. In this work, we first develop a distributed cognitive radio MAC (COMAC) protocol that enables unlicensed users to dynamically utilize the spectrum while limiting the interference on primary (PR) users. The main novelty in COMAC lies in not assuming a predefined CR-toPR power mask and not requiring active coordination with $P R$ users. COMAC provides a statistical performance guarantee for PR users by limiting the fraction of the time during which the PR users' reception is negatively affected by $C R$ transmissions. To provide such a guarantee, we develop probabilistic models for the PR-to-PR and the PR-to-CR interference under a Rayleigh fading channel model. From these models, we derive closedform expressions for the mean and variance of interference. Empirical results show that the distribution of the interference is approximately lognormal. Based on the developed interference models, we derive a closed-form expression for the maximum allowable power for a CR transmission. We extend the min-hop routing to exploit the available channel information for improving the perceived throughput. Our simulation results indicate that COMAC satisfies its target soft guarantees under different traffic loads and arbitrary user deployment scenarios. Results also show that exploiting the available channel information for the routing decisions can improve the end-to-end throughput of the CRN.
\end{abstract}

Index Terms-Opportunistic access, Power mask, Interference, Outage probability.

\section{INTRODUCTION}

Advances in wireless communication systems have significantly increased the demand for more transmission capacity. The unlicensed portions of the frequency spectrum (e.g., the ISM bands) have become increasingly crowded. At the same time, the FCC has recently reported that licensed bands are vastly underutilized [1], [2]. To overcome spectrum scarcity, licensed spectrum bands need to be more intelligently utilized. For this purpose cognitive radios (which are based on programmable-radio platforms) have been proposed to allow opportunistic spectrum access for unlicensed users.

A CRN has unique characteristics that distinguishes it from a traditional wireless communication network. The latter allocates spectrum statically, resulting in spectrum wastage,

This research was supported in part by NSF (under grant CNS0721935), Raytheon, and the Connection One center (an I/UCRC NSF/industry/university consortium). Any opinions, findings, conclusions, or recommendations expressed in this paper are those of the author(s) and do not necessarily reflect the views of the National Science Foundation. and has a fixed radio functionality. In contrast, a CRN dynamically utilizes the available spectrum and adapts its operating parameters (e.g., carrier frequency, number of channels to use, etc.) according to the surrounding environment [3], [4]. In an environment where several licensed primary radio networks (PRNs) are operating, a network of CR users that co-exist with PR users needs to exploit the underutilized portion of the spectrum. In this case, the crucial challenge is how to allow CR users to share the licensed spectrum with PR users without degrading the performance of the PR users. In this paper, we advocate a statistical approach by which CR users are allowed to communicate opportunistically while probabilistically guaranteeing the performance of PR users. Our key performance measure is the "outage probability" $\left(p_{\text {out }}\right)$ of a PR user, defined as the fraction of time during which the total interference power at a PR receiver exceeds the maximum tolerable interference.

The contributions of this paper are as follows.

- We develop stochastic models for the PR-to-PR and the PR-to-CR interference under a Rayleigh fading channel model. In the course of constructing these models, we derive closed-form expressions for the mean and variance of the total interference at a receiving node. Closed-form expressions for the characteristic function $(\mathrm{CF})$ of such interference are also obtained for integer-valued path loss exponents. Numerical and simulation results indicate that the resulting distribution of the total interference is well approximated by a lognormal function.

- We derive an expression for the maximum allowable powers for a CR transmission based on the developed models. The computed powers provide a statistical guarantee on the PRN performance.

- We design a distributed CSMA/CA-based MAC protocol for CRNs (COMAC) that does not require online interaction with PRNs. Through a local exchange of control messages, COMAC enables a pair of CR users to select the minimum number of channels to use according to the surrounding interference and the rate demand of the CR transmitter. CR users can communicate over both unused and partially used licensed channels without needing to coordinate with PRNs. Most importantly, COMAC functions without assuming a predefined CR-to-PR interference power mask. For a given PR spectrum band, the power mask is defined as the maximum permissible 
transmission power of a CR user over that band. This mask is needed to ensure that the CR transmission does not cause unacceptable interference to neighboring PR users operating on the same band.

- We implement a channel-aware routing (CAR) mechanism for CRNs that extends the well-known minimum hop routing (min-hop) approach for improving the perceived throughput.

It should be noted that interference modeling in wireless networks was previously studied under the assumption of an infinite user population, operating within an unbounded field. For example, [5]-[7] assumed that nodes are distributed according to a Poisson distribution, and characterized the distribution of the interference for an idealized infinite-size network operating within an infinite field. No multipath fading was considered. It is easy to show that their model leads to total interference whose mean and variance are infinite. Such a model cannot be applied in our work, as we consider a finite number of users.

The rest of the paper is organized as follows. The related previous work is reviewed in Section II. Section III introduces our system model and assumptions. In Section IV, we develop stochastic models for the PR-to-PR and PR-to-CR interference, and verify these models. Section $V$ shows how to provide a statistical guarantee on the performance of PR users. We introduce our proposed MAC protocol in Section VI, and evaluate its performance in Section VII. Finally, Section VIII gives concluding remarks.

\section{RELATED WORK}

One of the key challenges to enabling CR communications is how to perform opportunistic medium access control while limiting the interference imposed on PR users. Recently, several attempts were made to develop MAC protocols for CRNs (e.g., [8]-[16]). Existing work on spectrum sharing/access protocols can be classified according to their architecture (centralized or decentralized), spectrum allocation behavior (cooperative or non-cooperative), and spectrum access technique (overlay or underlay) [2]. The IEEE 802.22 working group is in the process of standardizing a centralized MAC protocol that enables spectrum reuse by $\mathrm{CR}$ users operating on the TV broadcast bands [17]. In [12]-[14] centralized protocols were proposed for coordinating spectrum access. For an ad hoc CRN without centralized control, it is desirable to have a distributed MAC protocol that allows every CR user to individually access the spectrum.

DC-MAC [18] is a cross-layer distributed scheme for spectrum allocation/sensing. It provides an optimization framework based on partially observable Markov decision processes, with no insights into protocol design, implementation and performance. In [19], the authors proposed a decentralized channel-sharing mechanism for CRNs based on a gametheoretic approach under both cooperative and non-cooperative scenarios. However, they did not propose an operational MAC protocol. No guarantee on the performance of PRNs was considered.

The FCC defined the interference temperature model [3], which provides a metric for measuring the interference expe- rienced by PR users. Clancy [20] used this model to select an optimal bandwidth/power assignment for CR users. However, no operational protocol was proposed. It is worth mentioning that due to the lack of specific technical rules to implement the interference temperature model, the FCC has abandoned this model in 2007 [21]. Three spectrum sharing techniques were proposed and compared in [5]: spreading-based underlay, interference avoidance overlay, and spreading-based underlay with interference avoidance. The metric of interest in the comparison was $p_{\text {out }}$. The treatment did not provide guarantees on the performance of PR users. Furthermore, interference statistics were used assuming an unbounded region for outage probability analysis.

Before closing, we note that a number of multi-channel contention-based MAC protocols were previously proposed in the context of CRNs (e.g., [8]-[11]). The CRN MAC protocol in [8] jointly optimizes the multi-channel power/rate assignment, assuming a given power mask on CR transmissions. How to determine an appropriate power mask remains an open issue. DDMAC [9] is a spectrum-sharing protocol for CRNs that attempts to maximize the CRN throughput through a novel probabilistic channel assignment algorithm that exploits the dependence between the signal's attenuation model and the transmission distance while considering the prevailing traffic and interference conditions. AS-MAC [10] is a spectrum-sharing protocol for CRNs that coexist with a GSM network. CR users select channels based on the CRN's control exchanges and GSM broadcast information. Explicit coordination with the PRNs is required. In [16], the authors developed a spectrum aware MAC protocol for CRNs (CMAC). CMAC enables opportunistic access and sharing of the available white spaces in the TV spectrum by adaptively allocating the spectrum among contending users.

To the best of our knowledge, COMAC is the first CRN MAC protocol that provides a soft guarantee on the performance of PR users without assuming a predefined interference power mask.

\section{SySTEM MODEL}

We consider a hybrid network, consisting of $M$ different PRNs and one CRN. The $M+1$ networks co-exist within the same geographical space. Figure 1 shows a conceptual view of the networks under consideration, where a group of PDAs use CRs to exploit the under-utilized spectrum in a WiMAX network (PRN 1) and a network of cell phones (PRN 2). Figure 1 also shows the operating spectrum of such a hybrid environment.

The PRNs are licensed to operate on different, nonoverlapping frequency bands. PR users that belong to a given PRN share the same licensed spectrum. In reality, a PRN may occupy multiple, non-contiguous frequency bands. Such a PRN can be easily captured in our setup by using multiple virtual PRNs, each operating over its own band. For the $i$ th PRN, we denote its carrier frequency, channel bandwidth, and maximum transmission power by $f_{i}, B_{i}$, and $P_{t}^{(i)}$, respectively. To make our analysis tractable, we model the locations of users in the $i$ th PRN as a homogeneous Poisson random variable on a disk area of parameter (density) $\rho_{i}$. This model 
was previously used in [6]-[8], [20]. In our simulations, we relax this assumption and consider arbitrary deployment scenarios. Each user in the $i$ th PRN acts as an ON/OFF source. We define the "activity factor" $\alpha_{i}$ as the fraction of time that a user in the $i$ th PRN is ON [22]-[24]. Estimating the activity behavior of PR users was investigated in [23], [24]. Specifically, in [23], $\alpha_{i}$ was estimated by maintaining a run length of the idle/busy period for each channel. Whenever the idle duration is ended by a PR transmission, the run length is recorded in a circular buffer. For our purposes, we assume that a similar mechanism for estimating $\alpha_{i}$ is in place. In section VII, we evaluate the impact of inaccurately estimating $\alpha_{i}$.

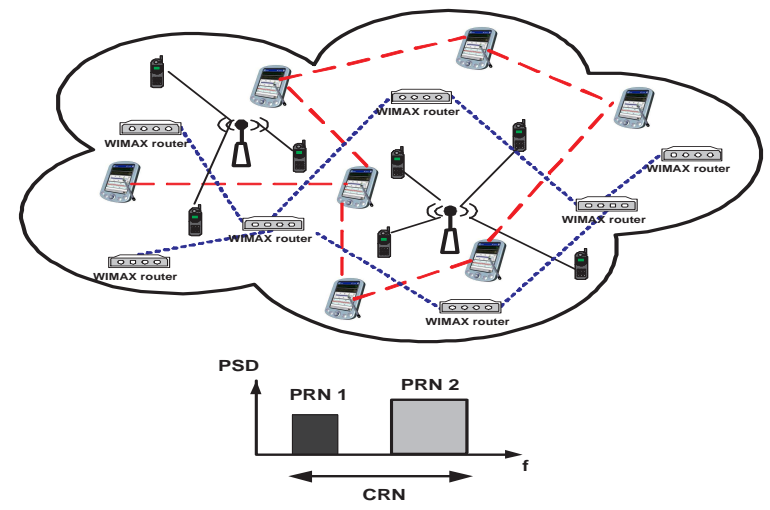

Fig. 1. A CRN environment containing one CRN of PDAs and 2 PRNs of WiMAX devices and cell phones.

CR users (unlicensed users) can opportunistically access the entire spectrum that is available to all PRNs. Each CR user is equipped with $n_{r}$ radio transceivers, $1 \leq n_{r} \leq M$, that can be used simultaneously. The CR user has a wideband sensing capability with a narrowband resolution. Such capability can be achieved using a wideband antenna, a power amplifier, and adaptive filters [2]. Thus, a CR user can sense the available spectrum in one shot (simultaneously sensing several GHzwide bands [25]) and estimate the instantaneous interference over each band. Such advanced spectrum sensing technology is readily available through a DSP technique called cyclostationary feature detection [2], [25], [26]. Alternatively, a sequential partial sensing approach can be employed at the cost of negligible switching/sensing overhead [11], [26]. It is worth mentioning that off-the-shelf wireless cards (e.g., ICS-572 products [27]) can readily serve as a fully functional wideband multi-channel CR interface. Such an interface enables a CR user to perform analysis of the RF spectrum (i.e., sensing) in real time. For a given CR transmission, the aggregate rate used over the assigned channels is fixed for the duration of that transmission. Our protocol assumes the availability of a pre-specified control channel of Fourier bandwidth $B_{c}$, where $B_{c} \ll B_{i}, i=1, \ldots, M$. Such a channel is not necessarily dedicated to the CRN. It may, for example, be one of the unlicensed ISM bands. Note that the existence of a dedicated common control channel is a characteristic of many MAC protocols proposed for CRNs (e.g., [8]-[10], [16], [18]).

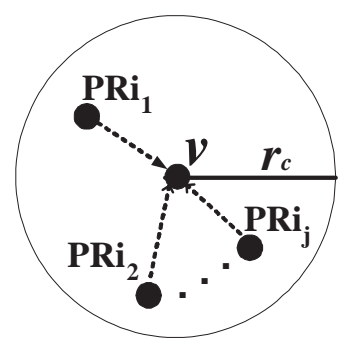

Fig. 2. Illustrating the aggregate interference from PRs at receiver $v$.

\section{INTERFERENCE ANALYSIS}

We develop stochastic models for the PR-to-PR and the PR-to-CR interference. Note that the $M$ different PRNs are licensed to operate over non-overlapping frequency bands (orthogonal bands). This fact ensures that the interference measured at a PR receiver over a band is only due to transmitters operating on that band (no mutual interference between different PRNs). Thus, without loss of generality, we consider one of the PRNs (PRN $i)^{1}$, where $i=1,2, \ldots, M$, and determine the total interference at a receiver $v$ (primary or cognitive) from only the PR users of that PRN (see Fig. 2). Let $d_{o}{ }^{(i)}$ be the close-in distance for the $i$ th PRN, defined as the distance from a transmitter after which the RF channel can be approximated by the free-space model [28]. Because of the highly nonlinear attenuation behavior of typical RF channels, we assume that the interference contributed to $v$ by PR users that lie outside a disk of radius $r_{c}\left(r_{c} \gg d_{o}^{(i)}\right)$ is negligible. This is inline with [29], in which $r_{c}$ was used to indicate the distance of the "first-tier interferers". Our simulations (Section VII) relax this assumption and account for all sources of interference, including those that are very far away from the receiver.

\section{A. Wireless Channel Model}

We consider a Rayleigh fading model to describe the channel between any two users. Specifically, for a transmitterreceiver separation $d$, the received power over the $i$ th channel ${ }^{2}$ is given by:

$$
P_{r}^{(i)}=P_{o}^{(i)}\left(\frac{d}{d_{o}{ }^{(i)}}\right)^{-n} \xi^{(i)}, \quad d \geq d_{o}^{(i)}
$$

where $P_{o}^{(i)}=\frac{P_{t}^{(i)} G_{t}^{(i)} G_{r}^{(i)} l_{i}^{2}}{\left(4 \pi d_{o}^{(i)}\right)^{2}}$ is the path loss of the closein distance $d_{o}^{(i)}, P_{t}^{(i)}$ is the transmission power, $G_{t}^{(i)}$ is the antenna gain of the transmitter, $G_{r}^{(i)}$ is the antenna gain of the receiver, $l_{i}$ is the wavelength of $f_{i}, n$ is the path loss exponent, and $\xi^{(i)}$ is a normalized random variable that represents the power gain of the fading process. For a Rayleigh fading, $\xi^{(i)}$ is exponentially distributed; $\operatorname{Pr}\left(\xi^{(i)} \leq y\right)=1-e^{-y}$ [28].

\footnotetext{
${ }^{1}$ Note that we consider a generic PRN. The derived expressions then apply to any of the $M$ PRNs by using the PRN's associated parameters (density, activity factor, etc.).

${ }^{2}$ Because of our assumed 1-to-1 mapping between the PRNs and the channels, the index $i$ is used to refer to either one.
} 
According to [28], $d_{o}{ }^{(i)}$ is given by $d_{o}{ }^{(i)}=$ $\max \left\{\frac{2 D^{2}}{l_{i}}, D, l_{i}\right\}$, where $D$ is the antenna length. In practice, $d_{o}{ }^{(i)}$ is of the same order of magnitude as the node's dimensions. For example, for a mobile phone operating at 900 MHz with $D=5 \mathrm{~cm}, d_{o}{ }^{(i)}=33 \mathrm{~cm}$. For an 802.11 WLAN card operating in the $2.4 \mathrm{GHz}$ band (5cm-long antenna), $d_{o}{ }^{(i)}=12 \mathrm{~cm}$. Accordingly, it is reasonable to assume that the probability that $d$ is less than $d_{o}{ }^{(i)}$ is very small.

\section{B. PR-to-CR Interference}

We now derive the statistics of the aggregate PR interference $P_{P R-C R}^{(i)}$ on a given CR receiver. Approximately, this is equal to the sum of the interference powers of all active PR transmitters within radius $r_{c}$ of the $\mathrm{CR}$ receiver, i.e., $P_{P R-C R}^{(i)} \approx \sum_{j} P_{r, j}^{(i)}$, where $P_{r, j}^{(i)}$ is the received power associated with the $j$ th active PR transmitter of the $i$ th channel, and the summation is carried out over all active PR transmitters in $R_{c}$.

Before proceeding further, we need to determine the distribution of the distance between a PR transmitter and a CR/PR receiver. Let $K_{i}$ denote the number of potential PR interferers within a disk area $R_{c}$, where $R_{c}=\pi r_{c}^{2}$. Since the locations of the PR users are modeled as a homogeneous Poisson process, the probability of $K_{i}=k_{i}$ is given by:

$$
\operatorname{Pr}\left\{K_{i}=k_{i}\right\}=\frac{e^{-\rho_{i} R_{c}}\left(\rho_{i} R_{c}\right)^{k_{i}}}{k_{i} !}, \quad k_{i}=0,1,2, \ldots
$$

The distribution of the locations of the $k_{i}$ interferers is that of $k_{i}$ independent and identically distributed (i.i.d.) uniform random variables ${ }^{3}$ [6]. Thus, the pdf of the distance $r$ between a receiver at the center of $R_{c}$ and an interferer that is randomly located inside $R_{c}$ is given by [6]:

$$
f_{R}(r)= \begin{cases}\frac{2 r}{r_{c}^{2}}, & r \leq r_{c} \\ 0, & \text { otherwise. }\end{cases}
$$

We assume that different interfering transmissions experience i.i.d. fades. This assumption is justified by noting that the distance between any two PR interferers is typically much larger than the wavelength of the carrier frequency of a PRN (e.g., for a PRN operating at $900 \mathrm{MHz}, l_{i}=33 \mathrm{~cm}$ ). Let $P_{r, j \mid y}^{(i)}$ denote the received power $P_{r, j}^{(i)}$ conditioned on $\xi_{j}^{(i)}=y$. Because the probability that the distance between a PR user and a CR user is less than $d_{o}{ }^{(i)}$ is approximately zero, the characteristic function $(\mathrm{CF})$ of $P_{r, j \mid y}^{(i)}$ can be written as:

$$
\begin{aligned}
\phi_{P_{r, j \mid y}^{(i)}}(\omega) & \stackrel{\text { def }}{=} E\left[e^{j \omega P_{r, j}^{(i)} \mid \xi_{j}^{(i)}}=y\right] \\
& \approx \int_{d_{o}^{(i)}}^{r_{c}} e^{j \omega P_{o}^{(i)}\left(\frac{r}{d_{o}^{(i)}}\right)^{-n} y} f_{R}(r) d r .
\end{aligned}
$$

By substituting (3) into (4) and algebraically manipulating the result, we obtain:

$$
\phi_{P_{r, j \mid y}^{(i)}}(\omega)=2\left(\frac{d_{o}^{(i)}}{r_{c}}\right)^{2} \int_{1}^{\frac{r_{c}}{d_{o}^{(i)}}} x e^{j \omega P_{o}^{(i)} x^{-n} y} d x .
$$

\footnotetext{
${ }^{3}$ In our simulations (Section VII), we study the performance of our protocol under both uniform and skewed user distributions.
}

The CF of $P_{r, j}^{(i)}, \phi_{P_{r, j}^{(i)}}(\omega)$, can be obtained by removing the conditioning in (5) and algebraically manipulating the result, leading to:

$$
\begin{aligned}
\phi_{P_{r, j}^{(i)}}(\omega) & =\int_{0}^{\infty} \phi_{P_{r, j \mid y}^{(i)}}(\omega) f_{\xi_{j}^{(i)}}(y) d y \\
& =\int_{0}^{\infty} \phi_{P_{r, j \mid y}^{(i)}}(\omega) e^{-y} d y \\
& =2\left(\frac{d_{o}^{(i)}}{r_{c}}\right)^{2} \int_{1}^{\frac{r_{c}}{d_{o}^{(i)}}} \frac{x^{n+1}}{x^{n}-j w P_{o}^{(i)}} d x .
\end{aligned}
$$

Recall that the number of PR users in the plane is Poisson distributed with mean of $\rho_{i}$ users per unit area. Because each PR user behaves as an ON/OFF source with activity factor $\alpha_{i}$, the number of active PR transmitters in $R_{c}$ (denoted by $N_{i}$ ) forms a Poisson random variable with mean of $\alpha_{i} \rho_{i}$ active users per unit area. Conditioned on $N_{i}=n_{i}$, the CF of $P_{P R-C R}^{(i)}$ is given by:

$$
\begin{aligned}
& E\left[e^{j \omega P_{P R-C R}^{(i)} \mid N_{i}}=n_{i}\right] \approx\left(\phi_{P_{r, j}^{(i)}}(\omega)\right)^{n_{i}} \\
& =\left(2\left(\frac{d_{o}^{(i)}}{r_{c}}\right)^{2} \int_{1}^{\frac{r_{c}}{d_{o}^{(i)}}} \frac{x^{n+1}}{x^{n}-j w P_{o}^{(i)}} d x\right)^{n_{i}} .
\end{aligned}
$$

The CF of the total PR-to-CR interference over channel $i$, $\phi_{P_{P R-C R}^{(i)}}(\omega)$, can be obtained by removing the conditioning in (7):

$$
\begin{aligned}
\phi_{P_{P R-C R}^{(i)}}(\omega) & =\sum_{n_{i}=0}^{\infty} \frac{e^{-\alpha_{i} \rho_{i} R_{c}}\left(\alpha_{i} \rho_{i} R_{c}\right)^{n_{i}}}{n_{i} !} \\
& \times\left(2\left(\frac{d_{o}^{(i)}}{r_{c}}\right)^{2} \int_{1}^{\frac{r_{c}}{d_{o}^{(i)}}} \frac{x^{n+1}}{x^{n}-j \omega P_{o}^{(i)}} d x\right)^{n_{i}} .
\end{aligned}
$$

Observe that the integral term in (8), denoted by $I_{C R, i}(n, \omega)$, is a function of $n, \omega$, and $f_{i}$. Thus, by summing the series, (8) can be rewritten as:

$$
\begin{aligned}
& \phi_{P_{P R-C R}^{(i)}}(\omega)= \\
& \exp \left(\alpha_{i} \rho_{i} R_{c}\left[\left(2\left(\frac{d_{o}^{(i)}}{r_{c}}\right)^{2} I_{C R, i}(n, \omega)\right)-1\right]\right) .
\end{aligned}
$$

For integer values of $n, I_{C R, i}(n, \omega)$ has a closed-form solution, and thus, the CF in (9) has a closed-form expression. As an example, the expression of $I_{C R, i}(4, \omega)$ is given by:

$$
\begin{array}{r}
I_{C R, i}(4, \omega)=\frac{1}{2}\left[\left(\frac{r_{c}}{d_{o}^{(i)}}\right)^{2}-1\right]+j^{\frac{3}{2}} \frac{\sqrt{\omega P_{o}^{(i)}}}{2} \times \\
{\left[\tan ^{-1}\left(\sqrt{\frac{j}{\omega P_{o}^{(i)}}}\left(\frac{r_{c}}{d_{o}^{(i)}}\right)^{2}\right)-\tan ^{-1}\left(\sqrt{\frac{j}{\omega P_{o}^{(i)}}}\right)\right] .}
\end{array}
$$

For the case of a non-integer $n, I_{C R, i}(n, \omega)$ can be evaluated numerically.

Using the fact that $\frac{d_{o}^{(i)}}{r_{c}} \ll 1$, we arrive at the following ap- 
proximate expressions for the mean and variance of $P_{P R-C R}^{(i)}$ :

$$
\begin{aligned}
& \bar{P}_{P R-C R}^{(i)} \stackrel{\text { def }}{=} \mathrm{E}\left[P_{P R-C R}^{(i)}\right] \stackrel{\text { def }}{=} \phi_{P_{P R-C R}^{(i)}}^{\prime}(0) \approx \\
& \begin{cases}\frac{2 \pi \alpha_{i} \rho_{i} P_{o}^{(i)} d_{o}^{(i) 2}}{(2-n)} e^{-\pi \alpha_{i} \rho_{i} d_{o}^{(i) 2}}\left[\left(\frac{r_{c}}{d_{o}^{(i)}}\right)^{2-n}-1\right], & 1 \leq n<2 \\
2 \pi \alpha_{i} \rho_{i} P_{o}^{(i)} d_{o}^{(i)^{2}} e^{-\pi \alpha_{i} \rho_{i} d_{o}^{(i)^{2}}} \ln \left[\frac{r_{c}}{d_{o}^{(i)}}\right], & n=2 \\
\frac{2 \pi \alpha_{i} \rho_{i} P_{o}^{(i)} d_{o}^{(i) 2}}{n-2} e^{-\pi \alpha_{i} \rho_{i} d_{o}^{(i) 2}}, & n>2\end{cases}
\end{aligned}
$$

and

$$
\begin{gathered}
\sigma_{P_{P R-C R}^{(i)}}^{2} \stackrel{\text { def }}{=} \operatorname{var}\left(P_{P R-C R}^{(i)}\right) \approx \\
\begin{cases}\frac{\pi \alpha_{i} \rho_{i}}{(n-1)}\left[2 P_{o}^{(i)} d_{o}^{(i)} e^{\left.-\pi \alpha_{i} \rho_{i} d_{o}^{(i)}\right)^{2}}\right]^{2}, & n>1 \\
2 \pi \alpha_{i} \rho_{i}\left[2 P_{o}^{(i)} d_{o}^{(i)} e^{\left.-\pi \alpha_{i} \rho_{i} d_{o}^{(i)}\right]^{2}}\right]^{2} \ln \left(\frac{r_{c}}{d_{o}^{(i)}}\right), & n=1 .\end{cases}
\end{gathered}
$$

Note that the above approximations for the mean and the variance show no dependence on $r_{c}$ for $n>2$.

While a closed-form expression for the pdf of $P_{P R-C R}^{(i)}$ cannot be found, numerical inversion of the $\mathrm{CF}$ and empirical fitting of the simulated data (Section IV-D) show that this pdf is well approximated by the lognormal distribution.

\section{PR-to-PR Interference}

In addition to estimating the PR-to-CR interference, our design requires a $\mathrm{CR}$ user to estimate the PR-to-PR interference so that an upper bound on the CR transmission power can be computed while providing a guarantee on $p_{\text {out }}$ for PR users. Let $b_{i}$ denote the minimum distance between a PR receiver and the nearest PR interferer ${ }^{4}$. This value is transmissiontechnology dependent and is fixed for a given PRN. For example, in a cellular network in which adjacent cells do not use common frequencies, $b_{i}$ is the minimum reuse distance, defined as the minimum distance between a base station of a cell and a mobile terminal of another non-adjacent cell that guarantees acceptable link quality. This value is easily shown to be equal to the diameter of a cell.

To characterize the PR-to-PR interference, we use a similar methodology to that used in the previous section. We replace the lower integration limit in (4) by $b_{i}$. The CF of PR-to-PR interference is thus given by:

$$
\begin{aligned}
& \phi_{P_{P R-P R}^{(i)}}(\omega)= \\
& \exp \left(\alpha_{i} \rho_{i} R_{c}\left[\left(2\left(\frac{d_{o}^{(i)}}{r_{c}}\right)^{2} I_{P R, i}(n, \omega)\right)-1\right]\right)
\end{aligned}
$$

where

$$
I_{P R, i}(n, \omega) \stackrel{\text { def }}{=} \int_{\frac{b_{i}}{d_{o}^{(i)}}}^{\frac{r_{c}}{d_{o}^{(i)}}} \frac{x^{n+1}}{x^{n}-j \omega P_{o}^{(i)}} d x .
$$

Consequently, the mean and variance for the PR-to-PR

\footnotetext{
${ }^{4}$ Mitigating interference over band $i$, and consequently achieving successful communications, necessitates imposing a minimum distance between active PR users.
}

interference are approximately given by:

$$
\begin{aligned}
& \bar{P}_{P R-P R}^{(i)} \approx \\
& \begin{cases}\frac{2 \pi \alpha_{i} \rho_{i} P_{o}^{(i)} d_{o}^{(i)} e^{2} e^{-\pi \alpha_{i} \rho_{i} b_{i}^{2}}}{(2-n)\left(d_{o}^{(i)}\right)^{2-n}}\left[r_{c}{ }^{2-n}-b_{i}{ }^{2-n}\right], & 1 \leq n<2 \\
2 \pi \alpha_{i} \rho_{i} P_{o}^{(i)} d_{o}^{(i)^{2}} e^{-\pi \alpha_{i} \rho_{i} b_{i}^{2}} \ln \left[\frac{r_{c}}{b_{i}}\right], & n=2 \\
\frac{2 \pi \alpha_{i} \rho_{i} P_{o}^{(i)} d_{o}^{(i)}{ }^{2}}{n-2} e^{-\pi \alpha_{i} \rho_{i} b_{i}^{2}}\left(\frac{b_{i}}{d_{o}^{(i)}}\right)^{2-n}, & n>2\end{cases}
\end{aligned}
$$

and

$$
\begin{aligned}
& \sigma_{P_{P R-P R}^{(i)}}^{2} \approx \\
& \begin{cases}\frac{\pi \alpha_{i} \rho_{i}}{(n-1)}\left[2 P_{o}^{(i)} d_{o}^{(i)} e^{-\pi \alpha_{i} \rho_{i} b_{i}^{2}}\right]^{2}\left(\frac{b_{i}}{d_{o}^{(i)}}\right)^{2(1-n)}, & n>1 \\
2 \pi \alpha_{i} \rho_{i}\left[2 P_{o}^{(i)} d_{o}^{(i)} e^{-\pi \alpha_{i} \rho_{i} b_{i}^{2}}\right]^{2} \ln \left(\frac{r_{c}}{b_{i}}\right), & n=1 .\end{cases}
\end{aligned}
$$

Similar to the case of PR-to-CR interference, for integer values of $n$, the $\mathrm{CF}$ in (13) has a closed-form expression. Furthermore, we found that the lognormal function well approximates the distribution of $P_{P R-P R}^{(i)}$.

\section{Model Verification}

We now use MATLAB simulations to empirically verify the validity of the derived PR-to-CR interference model (results for the validation of the PR-to-PR interference model are similar). We consider a circular field of radius 100 meters in which four PRNs are uniformly distributed. The transmission power for a PR user is 1 Watt. The antenna length $(D)$ is $5 \mathrm{~cm}$. Time is divided into slots. At any given slot, each user in PRN $i$ transmits with probability $\alpha_{i}$. Other PRN parameters are shown in Table I. We set $r_{c}=100 \mathrm{~m}$. First,

TABLE I

SIMULATION PARAMETERS USED TO VERIFY INTERFERENCE ANALYSIS.

\begin{tabular}{|c|c|c|c|}
\hline PRN & $f_{i}$ & $k_{i}$ & $\alpha_{i}$ \\
\hline 1 & $900 \mathrm{MHz}$ & 300 & 0.6 \\
2 & $1.5 \mathrm{GHz}$ & 400 & 0.5 \\
3 & $2.4 \mathrm{GHz}$ & 400 & 0.4 \\
4 & $4.0 \mathrm{GHz}$ & 200 & 0.2 \\
\hline
\end{tabular}

we assess the goodness of the approximations in (11) and (12) for the mean and variance of $P_{P R-C R}^{(i)}$. Table II shows the analytical approximations and the measured values for the means and variances, and the associated relative error $\left(\epsilon_{r}\right)$ when $n=4$ (similar behavior was observed for $n=2$ ). Each empirical value in the table is the average of 100 runs, each lasting for 100000 time slots. The results show that the derived expressions well approximate the measured statistics, with $\epsilon_{r}<1 \%$.

To validate the conjecture that the distribution of the interference model is well approximated by a lognormal distribution, we compute the pdf of the interference for PRN 1 with $n=2$ in two ways: by constructing the histogram of the simulated data and by numerically inverting the CF in (9). Figure 3(a) plots the empirical and numerically computed pdfs 
TABLE II

MEAN AND VARIANCE OF THE PR-TO-CR INTERFERENCE FOR $n=4$.

\begin{tabular}{|c||c|c|c|c|c|c|}
\hline \multirow{2}{*}{ PRN } & \multicolumn{3}{|c|}{ Mean } & \multicolumn{3}{c|}{ Variance } \\
\cline { 2 - 7 } & Analytical Approx. & Simulation & $\epsilon_{r}(\%)$ & Analytical Approx. & Simulation & $\epsilon_{r}(\%)$ \\
\hline \hline 1 & $1.2665 \times 10^{-5}$ & $1.2631 \times 10^{-5}$ & $0.2692 \%$ & $5.3468 \times 10^{-8}$ & $5.3804 \times 10^{-8}$ & $0.6245 \%$ \\
2 & $5.0661 \times 10^{-6}$ & $5.0202 \times 10^{-6}$ & $0.9135 \%$ & $2.1388 \times 10^{-8}$ & $2.1192 \times 10^{-8}$ & $0.9201 \%$ \\
3 & $1.5831 \times 10^{-6}$ & $1.5964 \times 10^{-6}$ & $0.8290 \%$ & $6.6836 \times 10^{-9}$ & $6.6408 \times 10^{-9}$ & $0.6436 \%$ \\
4 & $1.4248 \times 10^{-7}$ & $1.4309 \times 10^{-7}$ & $0.4263 \%$ & $6.0152 \times 10^{-10}$ & $5.9808 \times 10^{-10}$ & $0.5752 \%$ \\
\hline
\end{tabular}

of $P_{P R-C R}^{(1)}$ against the theoretical lognormal distribution with mean and variance given in (11) and (12), respectively. Visual inspection of the figure indicates the adequacy of the lognormal distribution. Figure 3(b) shows the probability plot of the

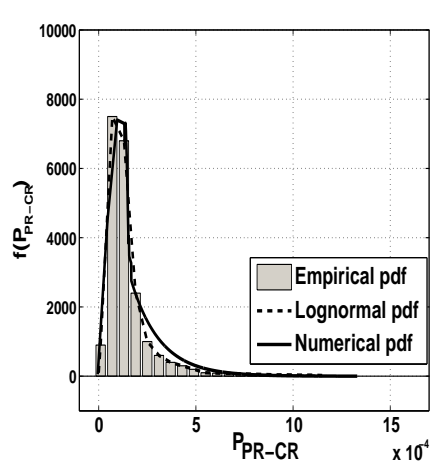

(a) Histogram

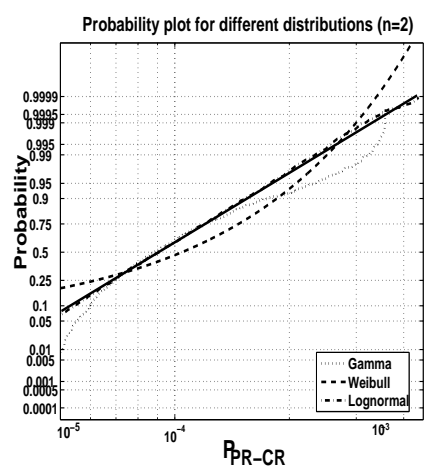

(b) Data-probability plot
Fig. 3. Interference model verification when $n=2$.

empirical data against three different distributions: Gamma, Weibull, and Lognormal ${ }^{5}$. In this figure, only the plotted points that correspond to the lognormal form reasonably straight lines and follow the empirical distribution fairly closely. Similar behaviors were observed for other PRNs and different values of $n$.

\section{Guaranteeing Outage Probability for PR USERS}

Because the outage probability $\left(p_{\text {out }}\right)$ is our primary performance metric, we statistically bound $p_{\text {out }}$ for each PRN. Our objective can be stated as follows: With probability $1-\beta$, where $\beta \ll 1$, the transmissions of $\mathrm{CR}$ users should not disturb the reception of any PR user. Therefore, we require that $p_{\text {out }} \leq \beta$. To provide such a guarantee, we need to compute the maximum allowable transmission power $P_{C, \beta}^{(i)}$ that a $\mathrm{CR}$ transmitter can use over channel $i$ such that all communicating PR users within the communication range of the transmitting $\mathrm{CR}$ are not impacted by this transmission with probability $1-\beta$. We enforce an exclusive channel occupancy policy on $\mathrm{CR}$ transmissions, whereby a channel occupied by a CR user

\footnotetext{
${ }^{5}$ The Data-probability plot is a graphical technique for assessing whether or not a data set follows a given distribution. If the probability plot approximately forms a straight line, then the distribution of the empirical data is well approximated by the suggested distribution.
}

cannot be simultaneously allocated to another CR user in the same vicinity (inline with the CSMA/CA mechanism). This policy ensures that the interference measured at a PR receiver is mainly due to at most one CR transmitter and to other PR transmitters. Accordingly, we compute an upper bound on the amount of interference that can be introduced by a CR transmitter over each channel. Consider the $j$ th PR user of the $i$ th PRN. With probability $1-\beta$, the following condition should be satisfied by every CR user:

$$
\begin{array}{r}
P_{P R-P R, j}^{(i)}+g_{C, j}^{(i)} P_{C, \beta}^{(i)} \leq P_{L}^{(i)}, \\
\forall j=1,2, \ldots, \text { and } \forall i=1,2, \ldots, M
\end{array}
$$

where $P_{P R-P R, j}^{(i)}$ is the total PR-to-PR interference power measured at the $j$ th PR receiver of the $i$ th PRN, $g_{C, j}^{(i)}$ is the gain between a CR transmitter and the $j$ th $\mathrm{PR}$ receiver, and $P_{L}^{(i)}$ is the interference power limit of a PR receiver in the $i$ th PRN. The value of $P_{L}^{(i)}$, which is sometimes referred to as the load or interference margin, is typically known for a given PRN (e.g., set by the FCC) [3], [20]. $P_{L}^{(i)}$ provides an upper bound on the potential interference that could be tolerated by a $P R$ receiver (i.e., defined at the $P R$ receiver not at the $C R$ transmitter). Consequently, using this interference limit, we derive the maximum permissible transmission power of a $\mathrm{CR}$ user such that a given outage probability is guaranteed.

Because we assume no active coordination between $\mathrm{CR}$ and PR users, $g_{C, j}^{(i)}$ is difficult to measure at a CR user. To proceed with our analysis, an estimate of $g_{C, j}^{(i)}$ that preserves the required bound on $p_{\text {out }}$ is needed to compute $P_{C, \beta}^{(i)}$. The problem of selecting $g_{C, j}^{(i)}$ was studied in [20], [30]. The $g_{C, j}^{(i)}$ was selected based on the shortest distance between a PR receiver and a CR transmitter. In [20], a probabilistic argument was used for computing the shortest distance. In [30], the shortest distance was derived from the spectrum sensing side information measured at a $\mathrm{CR}$ user.

We now derive the CDF of the distance between a $\mathrm{CR}$ transmitter and the closest active PR receiver. Based on such a CDF and following the same methodology in [20], we propose a mathematical formulation for selecting a value of $g_{C, j}^{(i)}$ that preserves the target $p_{\text {out }}$.

For a given PRN, let the distance between a CR transmitter located at the center of a disk of radius $r_{c}\left(r_{c} \gg d_{o}^{(i)}\right)$ and the closest active PR receiver be denoted by $R_{\min }$. Then, $R_{\min }=$ $\min \left\{R_{j}: j \in \Gamma\right\}$, where $R_{j}$ is a random variable representing the distance between a CR transmitter and the $j$ th PR receiver, and $\Gamma$ is the set of active PR receivers. According to (3), 
the CDF of $R_{j}$ is given by $F_{R_{j}}(r)=\frac{r^{2}}{r^{2}}$. Conditioning on given by: $N_{i}=n_{i}$, the CDF of $R_{\text {min }}$ is given by:

$$
\begin{aligned}
F_{R_{\min } \mid N_{i}=n_{i}}(r) & =1-\prod_{j=1}^{n_{i}}\left[1-F_{R_{j}}(r)\right] \\
& =1-\left[1-\left(\frac{r}{r_{c}}\right)^{2}\right]^{n_{i}} .
\end{aligned}
$$

Given that $N_{i}$ is a Poisson random variable (Section IV-B), the CDF of $R_{\text {min }}$ can be obtained by removing the conditioning in (18) and algebraically manipulating the result:

$$
F_{R_{\text {min }}}(r)=1-e^{-\alpha_{i} \rho_{i} \pi r^{2}} .
$$

Let $r^{*}$ denote the distance used in setting $g_{C, j}^{(i)}$ in (17). This $r^{*}$ can be selected based on a target percentage of $F_{R_{m i n}}$ (i.e., $\left.F_{R_{\text {min }}}\left(r^{*}\right)=1-p^{*}\right)$. Formally, with probability $p^{*}$, where $p^{*}$ is very close to one, the distance between a CR transmitter and its closest PR receiver is at least $r^{*}$. By substituting $F_{R_{\text {min }}}\left(r^{*}\right)=1-p^{*}$ in (19) and solving for $r^{*}$, we obtain:

$$
r^{*}=\sqrt{\frac{-\ln \left(p^{*}\right)}{\alpha_{i} \rho_{i} \pi}} .
$$

Depending on the relative location of a PR receiver with respect to a CR transmitter, there are two possible scenarios where outage can occur at a PR receiver:

1) The PR receiver falls within a distance less than $r^{*}$ from a CR transmitter. The likelihood of this scenario is $1-p^{*}$. In this case, we conservatively set $\operatorname{Pr}\left[\right.$ outage $\left.\mid r<r^{*}\right] \approx$ 1.

2) The PR receiver is at a distance greater than $r^{*}$ from a CR transmitter. The likelihood of this scenario is $p^{*}$. In this case, let $\gamma \stackrel{\text { def }}{=} \operatorname{Pr}\left[\right.$ outage $\left.\mid r>r^{*}\right]$.

Accounting for the above two scenarios, the overall outage probability can be computed via a straightforward application of Bayes's rule, i.e.,

$$
\begin{aligned}
p_{\text {out }}=\operatorname{Pr}\left[\text { outage } \mid r<r^{*}\right] \operatorname{Pr}\left[r<r^{*}\right]+ \\
\quad \operatorname{Pr}\left[\text { outage } \mid r>r^{*}\right] \operatorname{Pr}\left[r>r^{*}\right] \\
=1 \times\left(1-p^{*}\right)+\gamma \times p^{*}=1-(1-\gamma) p^{*} .
\end{aligned}
$$

Recall that we require $p_{\text {out }} \leq \beta$, which implies:

$$
\gamma \leq 1-\left(\frac{1-\beta}{p^{*}}\right)
$$

Note that $\gamma$ cannot be negative. Thus, for a valid bound on $\gamma$, the following constraint must be satisfied:

$$
\left(\frac{1-\beta}{p^{*}}\right)<1
$$

Equations (21) and (22) reveal that in order to preserve the required bound on $p_{\text {out }}$ (i.e., $\beta$ ), the condition in (17) should be satisfied by every CR user that is located at a distance greater than $r^{*}$, with probability $1-\gamma$. To satisfy this condition with probability $1-\gamma$, we compute the $(1-\gamma)$ quantile of $P_{P R-P R, j}^{(i)}$, denoted by $P_{\gamma}^{(i)}$. Because $P_{P R-P R}^{(i)}$ is approximately lognormally distributed, its $(1-\gamma)$-quantile is

$$
P_{\gamma}^{(i)}=e^{\left(\sigma_{P_{P R-P R}^{(i)}} \Phi^{-1}(1-\gamma)\right)}
$$

where $\Phi^{-1}$ is the $(1-\gamma)$-quantile of the standard normal distribution.

By substituting $P_{\gamma}^{(i)}$ in (17) and rearranging the equation, we obtain an upper bound on the interference that a CR transmitter is allowed to contribute to the $i$ th PRN while ensuring $p_{\text {out }} \leq \beta$ :

$$
P_{C, \beta}^{(i)} \leq \frac{P_{L}^{(i)}-P_{\gamma}^{(i)}}{g_{C, j}^{(i)}}, \quad i=1,2, \ldots, M .
$$

Accordingly, the maximum allowable transmission powers for a CR user over various channels are given by the vector $\vec{P}_{C, \beta}=\left[P_{C, \beta}^{(1)}, P_{C, \beta}^{(2)}, \ldots, P_{C, \beta}^{(M)}\right]$.

\section{THE COMAC PROTOCOL}

COMAC is a distributed and asynchronous MAC protocol for ad hoc CRNs that uses the previous analysis to enable opportunistic CR communications while providing soft guarantees on the performance of PR users. The proposed protocol uses a contention-based handshaking for exchange of control information. Before we describe the protocol's operation in detail, we first define and compute the different transmission regions around a CR user $A$. These regions describe $A$ 's "view" of its neighborhood.

\section{A. Transmission Regions for a CR User}

Each CR user $A$ is associated with a data region and a control region. Within these regions, other CR and PR users may exist. The data region of $A$ is defined as the area in which $A$ 's data transmission can be correctly decoded by another CR user. Let $r_{\text {data }}(A)$ be the radius of this region. With probability $1-\beta$, COMAC protects all PR receptions that are within distance $r_{\text {data }}(A)$ and that share channels with $A$. The control region of $A$ is defined as the region in which $A$ 's control packets can be correctly decoded. Let the radius of this region be $r_{c t r l}(A)$. As described below, COMAC requires $r_{c t r l}(A) \geq 2 r_{\text {data }}(A)$ to enforce exclusive channel occupancy among different $\mathrm{CR}$ transmissions.

In computing $A$ 's maximum transmission range over channel $i\left(a_{i}\right)$, we assume channel stationarity for the duration of one data packet. The received SINR at channel $i$ can be computed as:

$$
\mu_{i}=\frac{C\left(f_{i}\right) a_{i}^{-n} P_{A}^{(i)}}{\left(P_{t h}^{(i)}+P_{P R-C R}^{(i)}\right)}, \quad i=1, \ldots, M
$$

where $P_{t h}^{(i)}$ is the measured thermal noise over channel $i, P_{A}$ is $A$ 's transmission power, and $C\left(f_{i}\right)$ is a frequency-dependent constant, given by $C\left(f_{i}\right)=\frac{G_{t}^{(i)} G_{r}^{(i)} l_{i}^{2}}{(4 \pi)^{2}\left(d_{o}(i)\right)^{2-n}}$. According to COMAC, CR users transmit data packets using the maximum allowable power vector $\left(\vec{P}_{C, \beta}\right)$, derived in Section V. Using $\vec{P}_{C, \beta}$ and (26), the maximum distance at which a CR receiver can correctly decode $A$ 's data transmission over channel $i\left(a_{i}\right)$ 
is given by:

$$
a_{i}=\sqrt[n]{\frac{C\left(f_{i}\right) P_{C, \beta}^{(i)}}{\mu_{i}^{*}\left(P_{t h}^{(i)}+P_{P R-C R}^{(i)}\right)}}, \quad i=1, \ldots, M
$$

where $\mu_{i}^{*}$ is the SINR threshold required at the CR receiver to achieve a target bit error rate over channel $i$.

In (27), the CR-to-CR interference is ignored because of the aforementioned exclusive channel occupancy policy among CR users ${ }^{6}$. The PR-to-CR interference $P_{P R-C R}^{(i)}$ is estimated by its average expected interference at a $\mathrm{CR}$ transmitter, given in (11). Note that neighboring CR users typically experience similar average PR-to-CR interference [2], i.e., they share a similar view of the spectrum conditions. Thus, it makes little difference whether the parameters are computed at the transmitter or at the receiver.

Remark: In computing $a_{i}$, we do not consider the expected worst-case PR-to-CR interference since it typically leads to small control and data transmission ranges, which might jeopardize the network connectivity. On the other hand, ignoring the PR-to-CR interference results in longer control ranges, leading to over-conservative channel assignment and consequently lower CRN throughput.

Let $\mathcal{M}$ be the set of all $M$ channels. In COMAC, CR user A maintains a list of currently available channels, denoted by $\operatorname{LAC}(A)$, which consists of the channels in $\mathcal{M}$ that are not currently used by any of $A$ 's CR neighbors. $\operatorname{LAC}(A)$ is determined through the overheard control packets. We set $A$ 's data transmission range to:

$$
r_{\text {data }}(A)=\min _{j \in \operatorname{LAC}(A)} a_{j}
$$

We impose the following constraint on $r_{c t r l}(A)$ to control the CR-to-PR interference:

Proposition 1: If $r_{c t r l}(A) \geq 2 \max _{j \in \operatorname{LAC}(A)} a_{j}$, then there is no overlap between the data region of $A$ and the data region of any other CR transmitter that overlaps with $A$ in one or more data channels.

Proof. By definition, for any channel $j \in \operatorname{LAC}(A)$, $r_{\text {data }}(A) \leq a_{j}$ and $r_{c t r l}(A) \geq 2 a_{j}$. Because of the exclusive channel occupancy, within $r_{c t r l}(A)$ range no CR transmission other than $A$ 's can take place over channel $j$. Thus, the distance between $A$ and any other CR transmitter, say $C$, is at least $2 a_{j}$. If $C$ is outside the control region of $A$ and wants to reuse channel $j$, it will choose its $r_{\text {data }}(C)$ to be at most $a_{j}$. According to the proposition, $C$ will choose its $r_{c t r l}(C)$ to be at least $2 a_{j}$. Consequently, the data regions of $A$ and $C$ will not overlap, and only $A$ 's transmission will cause interference to PR users located in $A$ 's data region.

Remark: In general, the transmission range is a decreasing function of the transmission rate. Noting that the control channel requires a relatively low data rate, and consequently a low SINR threshold. Hence, the control range in Proposition 1 can be easily enforced through power control. Let $P_{c t r l}(A)$ be the minimum power level that is needed by a CR user $A$ to support

\footnotetext{
${ }^{6}$ Our simulations take into account the effect of the hidden-terminal problem due to imperfect control by considering the interference from active neighboring CR transmissions that use common channels (if any).
}

the range $r_{c t r l}(A)$ over the control channel. In computing $P_{c t r l}(A)$, we account for the channel-specific RF attenuation and interference behavior. Formally, we set $P_{c t r l}(A)=$ $\mu_{c}^{*} I^{(c)} /\left(C\left(f_{c}\right) r_{c t r l}(A)\right)$, where $f_{c}, \mu_{c}^{*}, C\left(f_{c}\right)$, and $I^{(c)}$ respectively denote the carrier frequency of the control channel, the SINR threshold required at the CR receiver to achieve a target bit error rate over the control channel, a frequencydependent constant $\left(C\left(f_{c}\right)=G_{t}^{(c)} G_{r}^{(c)} /(4 \pi)^{2} f_{c}^{2}\left(d_{o}^{(c)}\right)^{2-n}\right)$, and the average noise-plus-interference power over the control channel. To reduce the required value of $P_{c t r l}(A)$, the control channel should be selected in the lower portions of the spectrum, where higher transmission ranges can be achieved [16].

In Section VII, we study the impact of different settings of $r_{c t r l}(A)$ (as a function of $r_{\text {data }}(A)$ ) on the protocol's performance.

\section{B. Spectrum Access}

We propose a spectrum access mechanism that enables the $\mathrm{CR}$ transmitter and receiver to agree on the set of channels to use. This mechanism also ensures that with probability $1-\beta$ the ensuing data transmission will not disturb any of the PR users in the vicinities of the CR transmitter and receiver.

The spectrum access mechanism is described as follows. Suppose that CR user $A$ has data to transmit to CR user $B$ at a total data rate $R_{A}$. This $R_{A}$ is supported through the aggregate rate of all selected channels, i.e., $R_{A} \leq \sum_{i=1}^{m} r_{i}$, where $m$ is the number of channels that will be assigned to $A$ 's transmission, $m \leq n_{r}$, and $r_{i}$ is the data rate of the $i$ th selected channel. Let $\Omega(A, B)$ be the set of channels assigned to the transmission $A \rightarrow B$, where $m=\|\Omega(A, B)\|$. As described in Section VI-C, the selection of $\Omega(A, B)$ depends on spectrum state information (SSI). For now, it suffices to say that SSI includes the following information: (1) $\operatorname{LAC}(A, B) \stackrel{\text { def }}{=} \operatorname{LAC}(A) \cap \operatorname{LAC}(\mathrm{B})$; (2) the instantaneous interference level at $B$ over each channel in $\operatorname{LAC}(A, B)$; and (3) the channel gain between $A$ and $B$, computed using the received signal strength of $A$ 's control packets.

If $A$ does not sense a carrier over the control channel for a randomly selected backoff period, it computes its $r_{\text {data }}(A)$ according to (27) and (28). It then sends a Request-to-Send (RTS) message at power $P_{c t r l}(A)$ (computed according to Proposition 1). The RTS packet includes $\operatorname{LAC}(A), P_{\text {ctrl }}(A)$, $R_{A}$, and $P_{C, \beta}^{(i)}(A), \forall i \in \operatorname{LAC}(A)$. The neighbors of $A$, other than $B$, that can correctly decode the RTS will stay silent until either they receive another control packet, denoted by DCTS (explained below), or the expected time-out for that packet expires. Upon receiving the RTS packet, $B$ determines the SSI and proceeds with the channel assignment process, whose purpose is to determine whether or not there exists a feasible set of channels $\Omega(A, B) \subseteq \operatorname{LAC}(A, B)$ that can support the total traffic demand $R_{A}$. Depending on the outcome of the channel assignment process, $B$ decides whether or not $A$ can transmit. If not, then $B$ does not respond to $A$. Otherwise, $B$ sends a Clear-to-Send (CTS) message to $A$, which contains $\Omega(A, B)$ and the duration $\left(T_{p k t}(A)\right)$ needed to reserve the assigned channels for the ensuing data transmission 
and associated ACK packet. The CTS implicitly instructs the $\mathrm{CR}$ neighbors of $B$ to refrain from transmitting over the set of assigned channels for the duration $T_{p k t}(A)$. Once $A$ receives the CTS, it replies back with a "Decided-Channels-to-Send" (DCTS) message, informing its neighbors of $\Omega(A, B)$ and $T_{p k t}(A)$. After completing the RTS/CTS/DCTS exchange, the transmission $A \rightarrow B$ proceeds. Once completed, $B$ sends back an ACK packet to $A$ over the channel in $\Omega(A, B)$ that has the highest rate.

Because there is no interference between data and control packets, a CR that hears the RTS (CTS) packet defers its transmission only until the end of the control packet handshaking. This allows for more parallel transmissions to take place in the same vicinity. Before concluding this section, we give the formats of the various control packets. For a CR transmitter $A$ and a CR receiver $B$, the formats of the RTS, CTS, and DCTS are:

$$
\begin{aligned}
\operatorname{RTS}(A \rightarrow B) & =\left\{A, B, \operatorname{LAC}(A), P_{c t r l}(A), R_{A}, P_{C, \beta}^{(i)}(A)\right\} . \\
\operatorname{CTS}(B \rightarrow A) & =\left\{B, A, \Omega(A, B), T_{p k t}(A)\right\} . \\
\operatorname{DCTS}(A \rightarrow B) & =\left\{A, B, \Omega(A, B), T_{p k t}(A)\right\} .
\end{aligned}
$$

\section{Channel Assignment}

It is known that using the maximum possible channels for a transmission reduces the CR-to-PR interference [3], [8]. However, this may lead to channel over-assignment, which reduces the opportunity for assigning available channels to other CR transmitters [2], [8]. In our work, we statistically bound the CR-to-PR interference while using the minimum possible number of channels. Three parameters impact channel assignment: (1) the SSI, (2) $\vec{P}_{C, \beta}(A)$, and (3) $R_{A}$.

Based on the above parameters, receiver $B$ acts as follows:

- When $B$ receives $A$ 's $\mathrm{RTS}$, it first checks $\operatorname{LAC}(A, B)$ and removes any channel $i$ whose received SINR $\mu_{i}$ is less than $\mu_{i}^{*}$ (note that the transmission power and instantaneous interference are known at $B$ ).

- $B$ sorts the rest of the available channels in a descending order of their data rates, calculated according to the receiver SINR and any predefined rate-vs-SINR relationships (e.g., Shannon's equation, staircase function, etc.). It then iteratively picks channels from the top of the sorted list until either the aggregate rate is satisfied, the sorted list is exhausted (i.e., no feasible channel assignment can be found), or the number of selected channels exceeds $n_{r}$. In the latter two cases, $B$ will not respond to $A$ 's RTS, prompting $A$ to back off and retransmit later. It is easy to show that this channel assignment is optimal in terms of minimizing the number of selected channels. Algorithm 1 summarizes the channel assignment process.

Figure 4 depicts two scenarios for the operation of COMAC. In the first scenario (Figure 4(a)), the two transmitters $A$ and $C$ cannot hear each other's control packets. So, according to Proposition 1, the transmissions $A \rightarrow B$ and $C \rightarrow D$ can overlap in their data channels. In Figure 4(b), node $C$ falls in the control region of node $A$ (and vice versa). The exclusive channel occupancy policy prevents $A$ and $C$ from using common channels. However, the two transmissions can

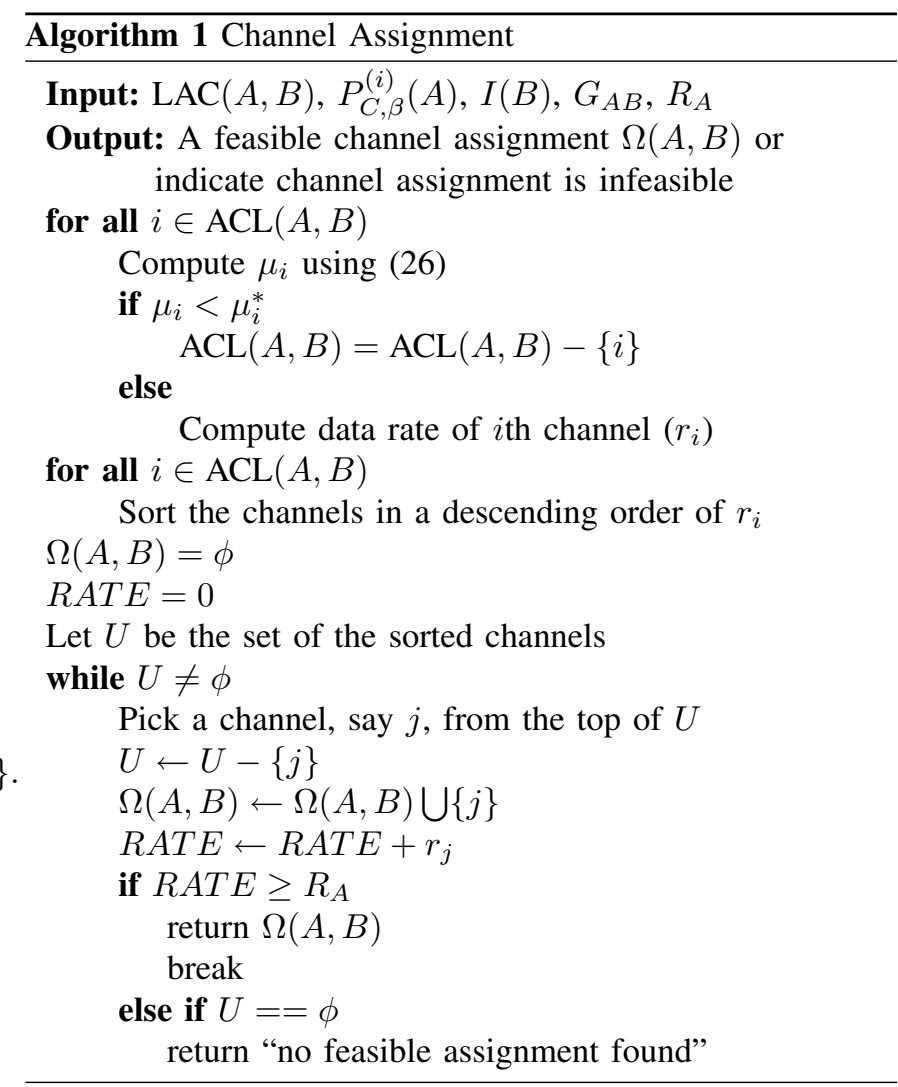

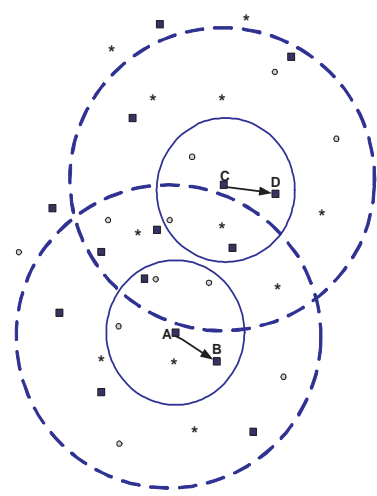

(a) Possible channel reuse

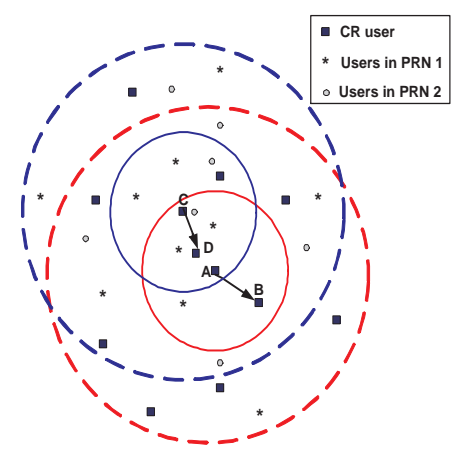

(b) Impossible channel reuse
Fig. 4. Scenarios in which a $C R$ transmitter $C$ can/cannot reuse the channels assigned to $A$. Solid circles indicate data-transmission ranges, while dashed circles indicate control-transmission ranges.

proceed simultaneously if $A$ and $C$ can find non-intersecting channels to support their rates.

\section{PERFormance Evaluation}

We now evaluate the performance of COMAC and study its effect on the performance of PR users. Our simulation programs are written in CSIM (a C-based process-oriented discrete-event simulation package) [31].

In our analysis, we assumed that the interference at a PR receiver is equal to the sum of the interference powers of all other interferers within an interference radius $r_{c}$. We also assumed that COMAC enforces an exclusive channel occupancy 


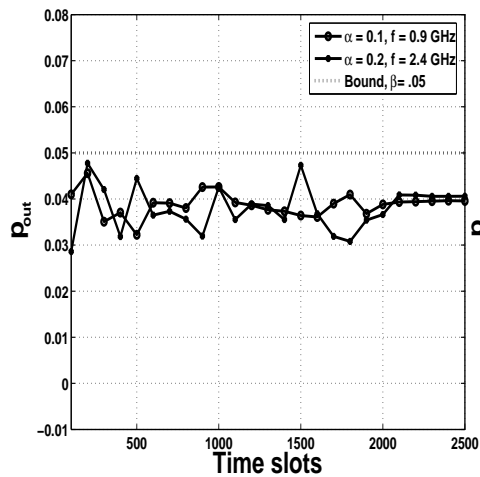

(a) $p_{\text {out }}$ vs. time $(\lambda=0.06)$

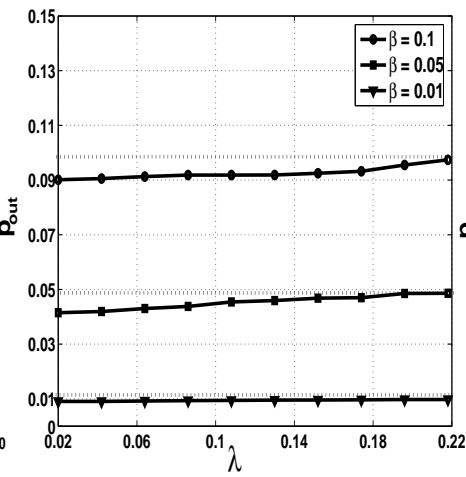

(b) $p_{\text {out }}$ vs. $\lambda$

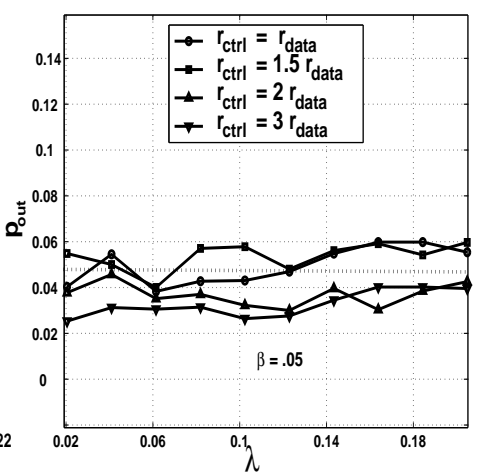

(c) $p_{\text {out }}$ (for different $\left.r_{c t r l}().\right)$ vs. $\lambda$

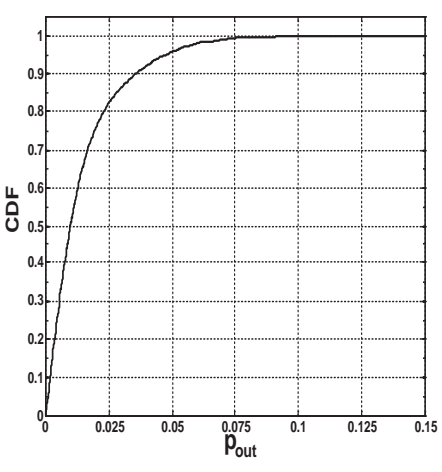

(d) CDF of observed $p_{\text {out }}(\beta=0.05)$

Fig. 5. Performance of a PRN.

policy on $\mathrm{CR}$ transmissions (i.e., no $\mathrm{CR}-\mathrm{CR}$ interference). Note, however, that hidden-terminal problem can occur in this scenario due to imperfect control. Our simulations relax these assumptions and account for all sources of interference, including those that are far away from a receiver (primary or cognitive) and use common channels. We focus on one-hop CR communications and investigate the effect of coexistence between the CRN and the PRNs on network performance. Our performance metrics include the outage probability for PR users, $p_{\text {out }}$, and the CRN goodput, defined as the average number of successfully received packets per time slot. For simplicity, we consider a fixed-packet size ( 2 Kbytes) and a fixed rate demand $\left(R_{A}=10 \mathrm{Mbps}\right)$ for all $\mathrm{CR}$ users. We set the control-packet size to 120 bits. A time slot corresponds to the transmission of one packet at $R_{A}$. We also measure the end-to-end goodput in multi-hop routing scenarios.

\section{A. Simulation Setup}

We simulate a system consisting of 8 PRNs and 1 CRN. Users in these networks are distributed over a $500 \times 500$ meters $^{2}$ area. We study both uniform and non-uniform node deployments. The first 4 PRNs operate in the $900 \mathrm{MHz}$ band, occupying 4 non-overlapping $2.5 \mathrm{MHz}$ channels with $P_{L}^{(i)}=2 \times 10^{-9} \mathrm{~W}$. The other 4 PRNs operate in the 2.4 $\mathrm{GHz}$ frequency band, occupying 4 non-overlapping $2.5-\mathrm{MHz}$ channels with $P_{L}^{(i)}=1 \times 10^{-10} \mathrm{~W}$. The activity factors for the 8 PRNs are $0.1,0.2,0.3,0.4,0.1,0.2,0.3$, and 0.4 , respectively. The number of PR users in each PRN is 200 . The transmission power for each PR transmitter is $1 \mathrm{~W}$ and the antenna length $(D)$ is $5 \mathrm{~cm}$. We set the minimum distance between a PR receiver and the nearest PR interferer $\left(b_{i}\right)$ to 25 meters for all $i$.

The CRN consists of 200 users. Each CR user generates packets according to a Poisson process with rate $\lambda$ (in packet/time slot), which is the same for all users. We set $n_{r}=4$; i.e., a CR user can use up to four data channels simultaneously. The signal propagation model in our simulations follows (1) with $n=4$. We set $\mu_{i}^{*}$ to $5 \mathrm{~dB}$ for all $i$. For all experiments, we select the value of $r^{*}$ (defined in Section V) such that $F_{R_{\min }}\left(r^{*}\right)=1-p^{*}=10^{-3}$. The reported results are averaged over 100 runs.

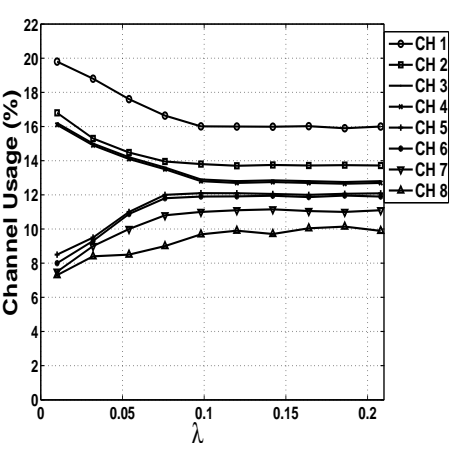

(a) CR channel usage vs. $\lambda$

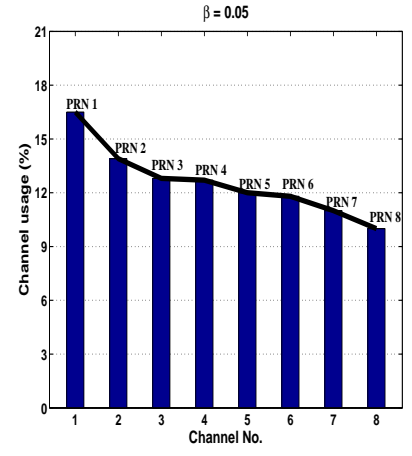

(b) CR channel usage (high load)
Fig. 6. Channel usage for the CRN.

\section{B. Single-hop Scenarios}

We first investigate the effect of CR transmissions on the performance of PR users assuming uniform node deployment. Figure 5(a) illustrates $p_{\text {out }}$ versus time ${ }^{7}$ for two PRNs (1 and 6 ) with $\beta=0.05$. The reported results are cumulative over time, i.e., 0-100, 0-200, 0-300, etc. It can be observed that $p_{\text {out }}$ is always less than $\beta=0.05$ for both PRNs. As time progresses, $p_{\text {out }}$ converges to a value less than 0.05 . These results are in line with the analysis in Section V.

For the next experiments, we focus on the performance for PRN 1 (other PRNs depicted similar behaviors). Figure 5(b) demonstrates $p_{\text {out }}$ as a function of $\lambda$ at $\beta=0.01,0.05$, and 0.1 . The results show that the bound on $p_{\text {out }}$ is always satisfied. The impact of different values of $r_{\text {ctrl }}($.$) on p_{\text {out }}$ is shown in Figure 5(c) with $\beta=0.05$. The figure illustrates that for $r_{c t r l}(.) \geq 2 r_{\text {data }}($.$) , the statistical guarantee is satisfied.$

\footnotetext{
${ }^{7}$ All figures reporting $p_{\text {out }}$ show only the PR user that experienced the highest interference among all PR users in the given PRN.
} 


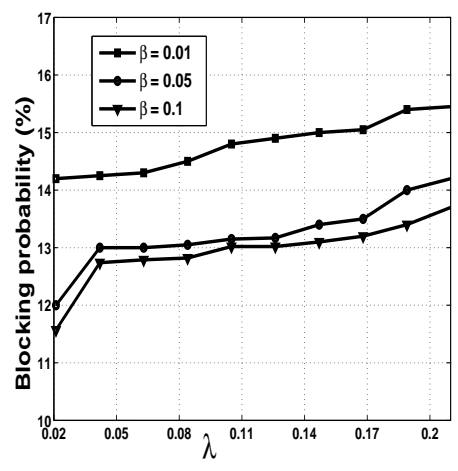

(a) CR blocking rate

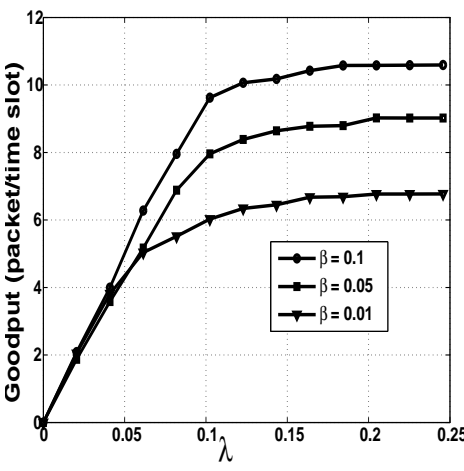

(b) CRN goodput for different values of $\beta$

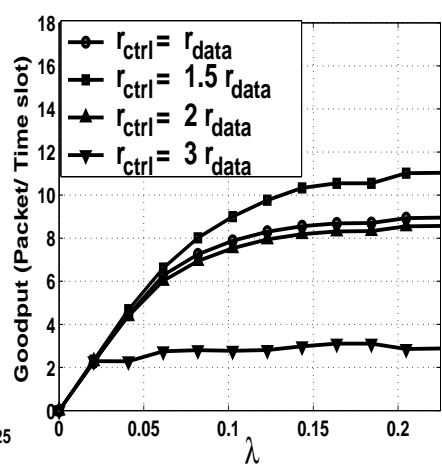

(c) $\mathrm{CRN}$ goodput for different values of $r_{\text {ctrl }}$

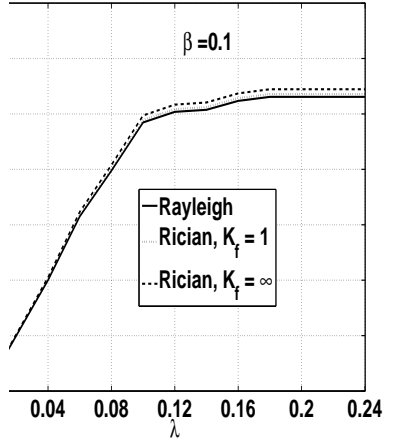

(d) Goodput vs. $\lambda$ (Rician and Raleigh channel models)

Fig. 7. Performance of the CRN.

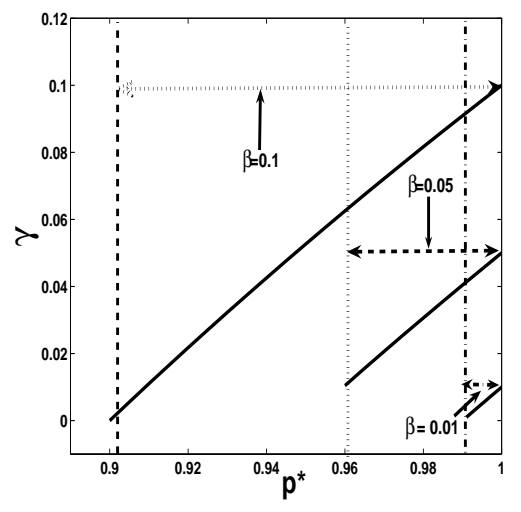

(a) $\gamma$ vs. $p^{*}$

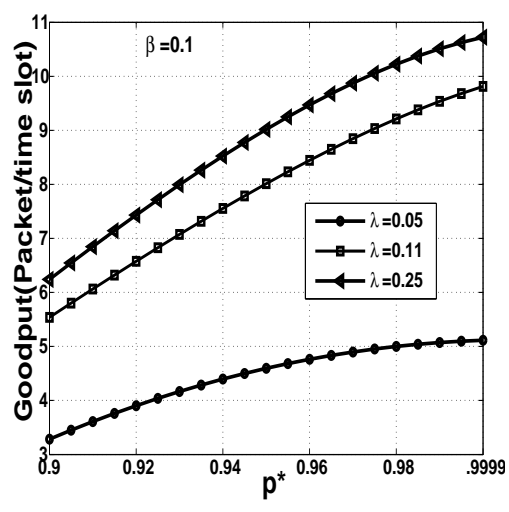

(b) Goodput vs. $p^{*}$

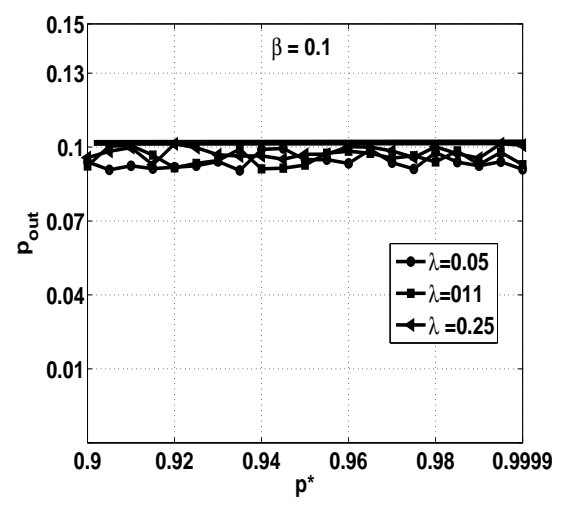

(c) $p_{\text {out }}$ vs. $p^{*}$

Fig. 8. Impact of selecting $p^{*}$.

However, for $r_{c t r l}()<.2 r_{\text {data }}($.$) , the statistical guarantee$ is not always satisfied. Figure 5(d) shows the CDF of the observed $p_{\text {out }}\left(F_{p_{\text {out }}}\right)$ with $\beta=0.05$. The figure reveals that $<5 \%$ of the time the total interference power at a $\mathrm{PR}$ receiver exceeds the maximum tolerable interference (i.e., $F_{p_{\text {out }}}(\beta=0.05)=\operatorname{Pr}\left[p_{\text {out }}<\beta\right]<0.95$.). Thus, the statistical guarantee is satisfied.

For a given $\beta$, Figures 6(a) and (b) depict the channel usage, defined as the fraction of time in which a specific channel is used for CR transmissions. These figures reveal that the carrier frequency and PRN activity factor affect channel usage (recall that the 8 PRNs differ in their $\alpha_{i}$ values). The smaller the value of $\alpha_{i}$, the higher is the utilization of channel by the CRN. The CRN utilization of the lower four channels is higher than that for the higher four channels (because of the lower attenuation). Even though channels with lower carrier frequencies and smaller activity factors are favored for CR transmissions (lower attenuation), under moderate and high traffic load, there are no significant differences in channel usage among all channels. Furthermore, channel usage remains fairly fixed in that traffic regime.
Figure 7(a) shows the effect of $\beta$ on the CRN connection blocking rate, defined as the fraction of CR packet attempts that need to back off due to channel unavailability. As demonstrated, the blocking probability is smaller at larger $\beta$. This is because a larger $\beta$ increases the maximum allowable powers for $\mathrm{CR}$ users, and consequently decreases the required number of channels to support the aggregate rate demand. Figure 7(b) indicates that a larger value of $\beta$ results in improved CRN goodput. This can be deduced from (24) and (25), as larger $\beta$ results in increasing $\vec{P}_{C, \beta}$. Consequently, each CR user can use fewer number of channels and more CR transmissions can take place concurrently. We study the impact of different control transmission ranges on the CRN goodput. Similar to the experiment in Figure 5(c), four control transmission radii are simulated: $r_{c t r l}=r_{\text {data }}, 1.5 r_{\text {data }}, 2 r_{\text {data }}$, and $3 r_{\text {data }}$. Figure 7(c) shows the CRN goodput as a function of packet generation rate under different values of $r_{c t r l}$ and a fixed $\beta=0.05$. The figure shows that increasing $r_{c t r l}$ (relative to $r_{\text {data }}$ ) has two conflicting consequences: (1) the transmission floor reserved by a CR user increases, and (2) the CR-to$\mathrm{CR}$ interference is reduced. The latter consequence reduces 


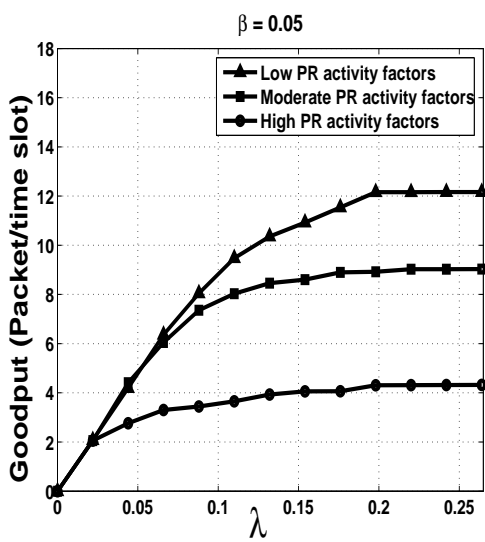

(a) CRN goodput for different $\alpha_{i}$ values

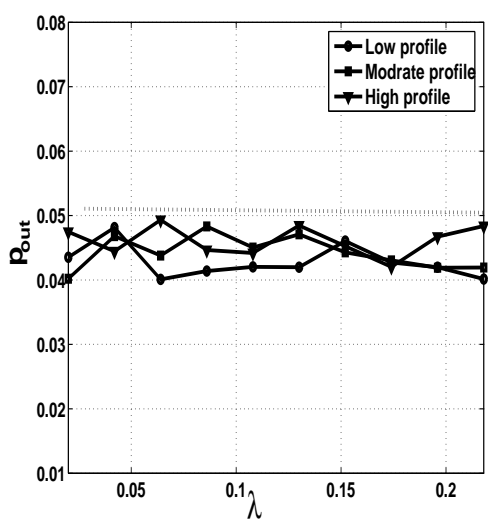

(b) $p_{\text {out }}$ vs. $\lambda$ (different $\alpha_{i}$ )

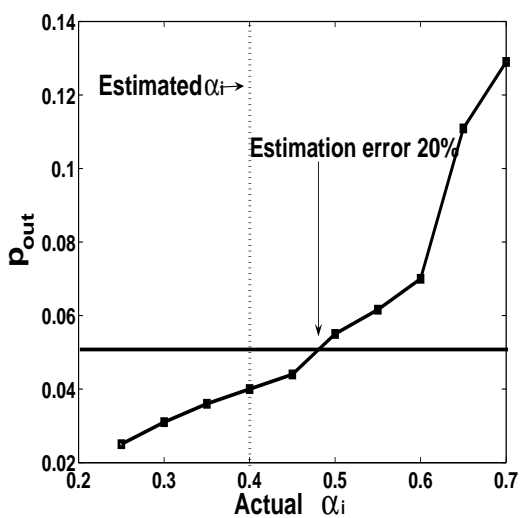

(c) $p_{\text {out }}$ under inaccurate $\alpha_{i}$

Fig. 9. Impact of activity profile on performance.

the mutual interference (i.e., improves goodput), while the former reduces the number of simultaneous transmissions (i.e., reduces goodput). Based on our simulations, we can draw the following observations: (1) for large values of $r_{c t r l}$, the first consequence dominates (i.e., there are fewer simultaneous $\mathrm{CR}$ transmissions). Consequently, the $\mathrm{CRN}$ goodput decreases with an increase in $r_{c t r l}$, (2) for relatively small values of $r_{c t r l}$ (e.g., between $r_{\text {data }}$ and $1.5 r_{\text {data }}$ ), we observe that the effect of the second consequence dominates, resulting in better goodput performance when $r_{c t r l}=1.5 r_{\text {data }}$ than when $r_{c t r l}=r_{\text {data }}$. The system performance under Raleigh and Rician channel models is investigated in Figure 7(d). Two Rician factors $\left(K_{f}\right)$ are simulated: $K_{f}=1$ and $\infty^{8}$. For a fair comparison, we considered normalized random variables to present the fading processes. The results show that both channel models give almost the same throughput performance. In both cases, the outage probability guarantee is still achieved.

We also investigate the effect of selecting $p^{*}$ (equivalently $r^{*}$ ) (defined in Section V) on the performance under different CR traffic loads. Figure 8(a) plots $\gamma$ over the feasible range of $p^{*}$ for different values of $\beta$. For $\beta=0.1$, Figures 8(a) and (b) indicate that using the largest possible value of $p^{*}$ (consequently the largest possible $\gamma$ ) results in improved CRN goodput. This can be deduced from (24) and (25), as larger $\gamma$ results in increasing $\vec{P}_{C, \beta}$ for CR users. The increase in $\vec{P}_{C, \beta}$ reduces the number of channels assigned to a CR transmitter, which allows for more concurrent transmissions. Figures 8(c) indicates that for all values of $p^{*}$ in Figure 8(b), the required guarantee is always achieved. Similar observations can be made for other values of $\beta$.

Next, we study the impact of $\alpha_{i}$ on performance. Three different activity profiles are simulated: high $\left(\alpha_{i}=0.8\right)$, moderate $\left(\alpha_{i}=0.4\right)$, and low $\left(\alpha_{i}=0.1\right)$. Figure 9(a) shows that the CRN goodput decreases for higher PRN activity profiles. Figure 9(b) indicates that the bound on $p_{\text {out }}$ is always achieved under different activity profiles. In Figure 9(c), we

\footnotetext{
${ }^{8}$ When $K_{f}=\infty$, the Rician channel reduces to the AWGN channel.
}

study the effect of inaccurately estimating $\alpha_{i}$. We operate COMAC assuming an estimated activity factor $\widetilde{\alpha_{i}}$ of 0.4 , and vary its actual value in the simulations. It is noted that the required bound on $p_{\text {out }}$ is not satisfied when $\alpha_{i}$ exceeds $\widetilde{\alpha_{i}}$ by more than $20 \%$. Thus, $\alpha_{i}$ has to be conservatively estimated.

Finally, we investigate the robustness of COMAC under a skewed user deployment. We assume that the network field is divided into four quadrants with respective PR user densities $60 \%, 25 \%, 10 \%$, and 5\%. Figure 10(a) illustrates that the required bound on $p_{\text {out }}$ is still satisfied. However, Figure 10(b) shows that a minor reduction in the CRN goodput may occur compared with a uniform node deployment.

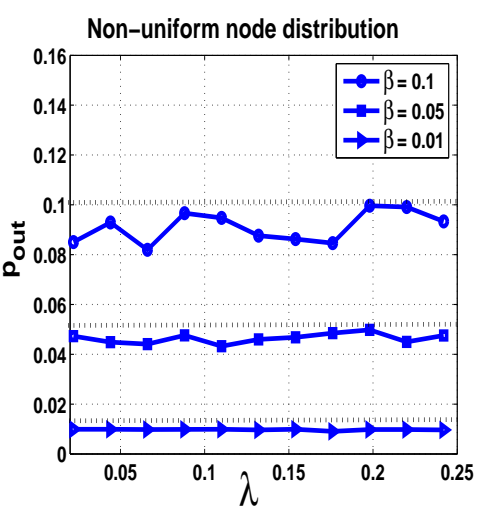

(a) $p_{\text {out }}$ vs. $\lambda$

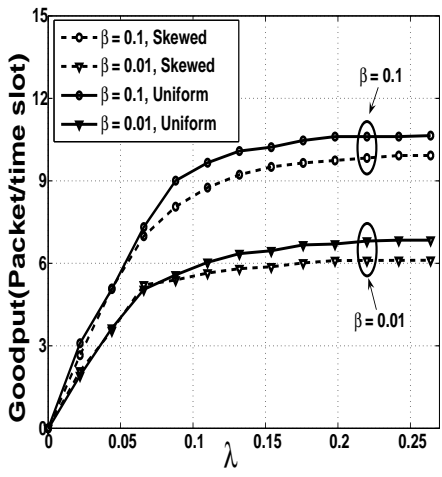

(b) CRN goodput for different $\beta$
Fig. 10. Performance under skewed and uniform user deployments.

\section{Multi-hop Scenarios}

We implement a channel-aware routing (CAR) mechanism for CRNs that extends the well-known minimum hop routing (min-hop) approach. We assume shortest path routing using Dijkstra's algorithm is used in the CRN network. CAR exploits the channel availability information to produce end-to-end 
routes that improve the end-to-end CRN goodput. It uses the hop count and the residual capacity of each link, defined as the maximum data rate a link can support over all the available channels. The selected route is one with the minimum number of hops that can support a given demand rate. This can be achieved by first pruning any link that has a residual capacity less than the source demand, then applying minhop routing on the pruned graph. The potential benefit of using CAR in contrast to min-hop routing is demonstrated in Figure 11, where a source $S$ attempts to transmit data to a destination $D$. Figure 11(a) shows node connectivity in the original graph. Min-hop routing always selects route $S-A-D$ for data transmissions, as shown in Figure 11(b). CAR will also use route $S-A-D$ if it satisfies the required rate. However, if some of the links cannot support the source demand, as demonstrated in Figure 11(c), CAR will select route $S-B-E$ $D$.

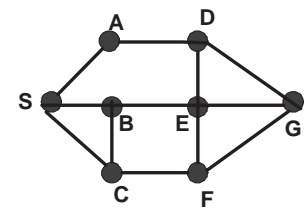

(a)

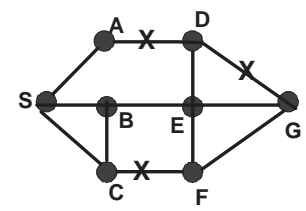

(c)

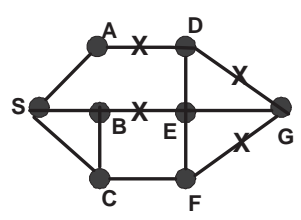

(e)

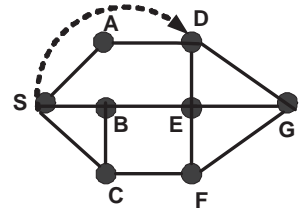

(b)

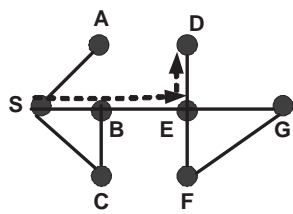

(d)

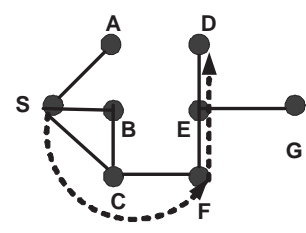

(f)
Fig. 11. Illustration of channel-aware routing.

We investigate the end-to-end goodput performance of COMAC as a function of the packet generation rate under min-hop routing and CAR. In our configuration, described above, we randomly select any pair of nodes as source and destination. For min-hop routing, the paths are computed only once at the beginning of the experiment. For CAR, we compute the route for every flow of packets between the source and destination. All the packets in that flow follow the same path. The measured average path length between any source and destination in our network was about seven hops. Figure 12 shows that CAR achieves up to $25 \%$ increase in the end-to-end goodput. This improvement is magnified under high loads.

\section{CONCLUSION}

In this paper, we proposed a MAC protocol for opportunistic CRNs. Our protocol, COMAC, improves spectrum utilization

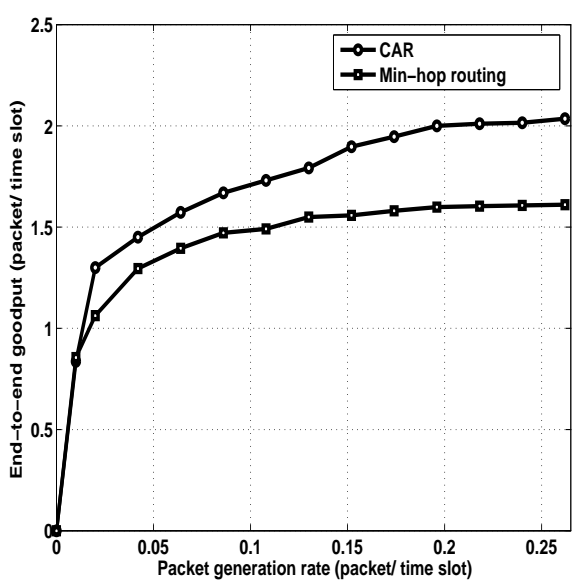

Fig. 12. End-to-end throughput of min-hop routing and CAR as a function of the packet generation rate.

while limiting the interference imposed on licensed users. We first developed stochastic models for the PR-to-PR and the PR-to-CR interference under a Rayleigh fading channel model, and derived closed-form expressions for the mean and variance of each interference component. Furthermore, closed-form expressions were obtained for the characteristic function of the total interference under typical path loss exponents. From the interference analysis, we derived a closedform expression for the maximum allowable powers for CR transmissions that ensure a statistical bound $\beta$ on $p_{\text {out }}$ for PR users. We integrated our theoretical analysis in the design of the COMAC protocol. Our simulation results showed that COMAC statistically guarantees the performance of PR users under different CR traffic loads and for different values of $\beta$. Results also showed that channel usage is reasonably balanced across various channels, even when the PR activity factors over such channels and the associated carrier frequencies are significantly different. Although uniform node deployment was used in our analysis, our simulations verified that the performance is not significantly impacted by the distributions of users in PRN/CRN. Finally, our simulation results showed that exploiting the available channel information for the routing decisions can improve the end-to-end throughput of the CRN by up to $25 \%$.

\section{REFERENCES}

[1] "FCC, spectrum policy task force report, ET docket no. 02-155," Nov. 2002.

[2] I. Akyildiz, W.-Y. Lee, M.C. Vuran, and S. Mohanty, "Next generation dynamic spectrum access cognitive radio wireless networks: A survey," Computer Networks, vol. 50, no. 13, pp. 2127-2159, 2006.

[3] S. Haykin, "Cognitive radio: Brain-empowered wireless communications," IEEE Journal on Selected Areas in Communications, vol. 23, no. 2, pp. 201-220, Feb. 2005.

[4] S. Hijazi, B. Natarajan, M. Michelini, Z. Wu, and C. Nassar, "Flexible spectrum use and better coexistence at the physical layer of future wireless systems via a multicarrier platform," IEEE Wireless Communications, vol. 11, no. 2, pp. 8-14, April 2004.

[5] R. Menon, R. Buehrer, and J. Reed, "Outage probability based comparison of underlay and overlay spectrum sharing techniques," in Proceedings of the IEEE DySPAN Conference, Nov. 2005, pp. 101-109. 
[6] E. Sousa and J. Silvester, "Optimum transmission ranges in a directsequence spread-spectrum multihop packet radio network," IEEE Journal on Selected Areas in Communications, vol. 8, no. 5, pp. 762-771, June 1990.

[7] E. Sousa, "Performance of a spread spectrum packet radio network link in a poisson field of interferers," IEEE Trans. Information Theory, vol. 38, pp. 1743-1754, Nov. 1992.

[8] T. Shu, S. Cui, and M. Krunz, "Medium access control for multi-channel parallel transmission in cognitive radio networks," in Proceedings of the IEEE GLOBECOM Conference, Nov. 2006.

[9] H. Bany Salameh, M. Krunz, and O. Younis, "Distance- and trafficaware channel assignment in cognitive radio networks," in Proceedings of the IEEE SECON Conference, June 2008.

[10] S. Sankaranarayanan, P. Papadimitratos, A. Mishra, and S. Hershey, "A bandwidth sharing approach to improve licensed spectrum utilization," in Proceedings of the IEEE DySPAN Conference, Nov. 2005, pp. 279288.

[11] Ashutosh Sabharwal, Ahmad Khoshnevis, and Edward Knightly, "Opportunistic spectral usage: Bounds and a multi-band CSMA/CA protocol," IEEE/ACM Transactions on Networking, vol. 15, no. 3, pp. 533545,2007

[12] V. Brik, E. Rozner, S. Banarjee, and P. Bahl, "DSAP: A protocol for coordinated spectrum access," in Proceedings of the IEEE DySPAN Conference, Nov. 2005, pp. 611-614.

[13] S. Zekavat and X. Li, "User-central wireless system: Ultimate dynamic channel allocation," in Proceedings of the IEEE DySPAN Conference, Nov. 2005, pp. 82-87.

[14] D. Cabric, S. Mishra, D. Willkomm, R. Brodersen, and A. Wolisz, "A cognitive radio approach for usage of virtual unlicensed spectrum," in Proceedings of the 14th IST Mobile and Wireless Communications Summit, June 2005.

[15] L. Ma, X. Han, and C.-C. Shen, "Dynamic open spectrum sharing MAC protocol for wireless ad hoc networks," in Proceedings of the IEEE DySPAN Conference, Nov. 2005, pp. 203-213.

[16] Y. Yuan, P. Bahl, R. Chandra, P. Chou, J. Ferrell, T. Moscibroda, S. Narlanka, and Y. Wu, "Knows: Kognitive networking over white spaces," in Proceedings of the IEEE DySPAN Conference, April 2007, pp. 416-427.

[17] "IEEE 802.22 Working Group on Wireless Regional Area Networks. http://www.ieee802.org/22/,"

[18] Q. Zhao, L. Tong, and A. Swami, "Decentralized cognitive MAC for dynamic spectrum access," in Proceedings of the IEEE DySPAN Conference, Nov. 2005, pp. 224-232.

[19] N. Nie and C. Comaniciu, "Adaptive channel allocation spectrum etiquette for cognitive radio networks," in Proceedings of the IEEE DySPAN Conference, Nov. 2005, pp. 269-278.

[20] T. C. Clancy, "Achievable capacity under the interference temperature model," in Proceedings of the IEEE INFOCOM Conference, May 2007, pp. 794-802.

[21] "Interference Temperature Operation FCC, ET Docket No. 03-237; FCC 07-78," 2007.

[22] S.N. Shankar, "Squeezing the most out of cognitive radio: A joint MAC/PHY perspective," in proceeding of Acoustics, Speech and Signal Processing (ASSP07), April 2007, pp. 1361-1364.

[23] C.-C. Hsu, D. Wei, and C.C.J. Kuo, "A cognitive MAC protocol using statistical channel allocation for wireless ad-hoc networks," in Proceedings of Wireless Communications and Networking Conference (WCNC07), March 2007, pp. 105-110.

[24] Yiping Xing, R. Chandramouli, Stefan Mangold, and Sai Shankar, "Dynamic spectrum access in open spectrum wireless networks," IEEE Journal on Selected Areas in Communications, vol. 24, no. 3, pp. 626637, 2006.

[25] D. Cabric and R. Brodersen, "Physical layer design issues unique to cognitive radio systems," in Proceedings of the IEEE International Symposium on Personal, Indoor and Mobile Radio Communications (PIMRC), Sept. 2005.

[26] D. Cabric, S. Mishra, and R. Brodersen, "Implementation issues in spectrum sensing for cognitive radios," in Proceedings of the ThirtyEighth Asilomar Conference on Signals, Systems and Computers, 2004, pp. 772-776.

[27] Application Note (AN-SR-7), "Building a versatile low latency cognitive radio for multi-mission applications with the ICS572," Tech. Rep., GE Fanuc Intelligent Platforms, Sep 2003, http://www.gefanucembedded.com/news-events/whitepapers/3067.

[28] T. S. Rappaport, Wireless Communications-Principles and Practice, Prentice-Hall Press, 2001, 2nd edition.
[29] C. Yu, K. G. Shin, and L. Song, "Link-layer salvaging for making routing progress in mobile ad hoc networks," in Proc. of the ACM International Symposium on Mobile and Ad-Hoc Networking and Computing (MobiHoc), May 2005, pp. 242-254.

[30] K. Hamdi, W. Zhang, and K. Ben Letaief, "Power control in cognitive radio systems based on spectrum sensing side information," in Proceedings of the IEEE ICC Conference, June 2007, pp. 5161-5165.

[31] "Mesquite Software Incorporation," www.mesquite.com.

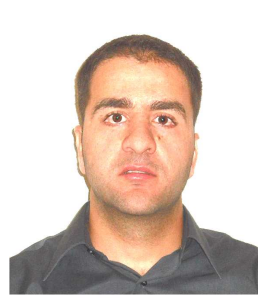

Haythem A. Bany Salameh received the B.Sc. and M.S. degrees in electrical engineering (with the highest distinction) from Jordan University of Science and Technology, Irbid, Jordan, in 2003 and 2005, respectively. In 2005, Yarmouk University, Jordan, awarded him a scholarship to pursue his study. He is currently working toward the Ph.D. degree in electrical and computer engineering at the University of Arizona, where he is a Research Assistant. His current research interests are in system architecture and communication protocol designs for wireless cognitive radio networks with emphasis on spectrum access and channel/power assignment. In Summer 2008, he was a member of the R\&D LTE (Long Term Evolution) Development Group, QUALCOMM, Inc., San Diego. He serves as a reviewer for many IEEE conferences and journals.

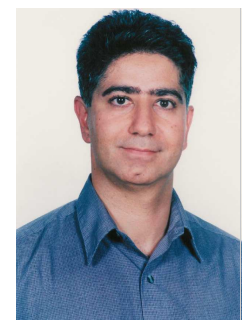

Marwan M. Krunz received the Ph.D. degree in electrical engineering from Michigan State University in July 1995. He joined the University of Arizona in January 1997, after a brief postdoctoral stint at the University of Maryland, College Park. $\mathrm{He}$ is currently a professor of electrical and computer engineering and the co-director of Connection One, a joint NSF/state/industry IUCRC cooperative research center that focuses on RF and wireless communication systems and networks. Dr. Krunz chaired the computer engineering group of the ECE Department (2006-2008). He previously held various visiting research positions at INRIA, HP Labs, US West Advanced Technologies, and Paris VI University. Dr. Krunz's research is in communications technology and networking, with particular emphasis on resource allocation, adaptive control, and distributed protocol design. Recently, he has been involved in projects related to cognitive radios ; distributed resource management in wireless networks; protocol design for ad hoc networks; MIMO and smart-antenna-based systems; UWB-based wireless personal area networks; energy management and clustering in sensor networks; video streaming; and fault detection in all-optical networks. He has published more than 140 journal articles and refereed conference papers in these areas. He received the NSF CAREER Award (1998-2002). He currently serves on the editorial board for the IEEE/ACM Transactions on Networking, the IEEE Transactions on Mobile Computing, and the Computer Communications Journal. He served as a Technical Program Chair for the IEEE INFOCOM 2004, the IEEE SECON 2005, and the IEEE WoWMoM 2006 Conferences.

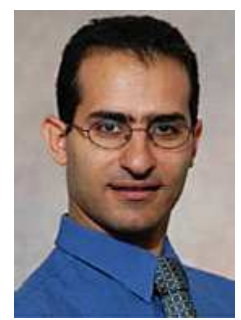

Ossama Younis is a senior research scientist in Applied Research at Telcordia Technologies, Inc. He received his B.S. and M.S. degrees in computer science from Alexandria University, Egypt. Then, he received his $\mathrm{Ph} . \mathrm{D}$. degree in computer science from Purdue University, USA in August 2005. He has served as the web chair of IEEE ICNP 2008 and on the Technical Program Committee of several international conferences. His research interests are in the architecture and experimentation of network protocols and applications, especially wireless sensor networks, cognitive-radio networks, and Internet tomography. Other interests include distributed systems, secure protocol design, and cross-layer optimization. He is a member of the ACM and the IEEE. 